\title{
Chromospheric Eruptions Observed with a Wide Slit
}

François Costard

22 av. Perichont, F-75016 Paris, France

\begin{abstract}
The method of placing the solar limb tangential to the slit, which is then widened, has been employed. The following phenomena have been observed: fast radial motion of low loops; extension of the main loop into the corona; ejection of material from the top of the loop; rotation of the structure as it relaxes.
\end{abstract}

\section{Reference}

Costard, F.: "L'eruption chromosphérique du 12/09/85", L'Astronomie, 1987 Jan., p.21-7

\section{Amateur/Professional Cooperation in 2 Solar Studies}

\section{T. Roudier, R. Muller, J.C. Hulot, and F.Vaissière}

Observatoire du Pic du Midi et de Toulouse, 14 av. Edouard Belin, F-31400 Toulouse, France

\begin{abstract}
The modification of properties of granules around magnetic flux tubes has been studied for the first time from photographs (at $\lambda=4803{ }^{\circ} \mathrm{A}$ and $5770 \AA$ ) taken with the $50-\mathrm{cm}$ refractor at Pic du Midi. Statistically, these granules are more numerous, smaller, and more elongated than other granules. During their first two minutes of life they show very pronounced radial orientation to the magnetic flux tube.

Angular measurements on the same granules have a precision of $\pm 10^{\circ}$, which is sufficient as theoretical studies show that they rotate by $360^{\circ}$ in the course of their life. Initial results appeared to show that only explosive granules had intrinsic rotation, but further examination showed that it is a general trend. It seems that the granules do rotate significantly, but that there is a more important "push-pull" effect, in agreement with A. Title's theory drawn from SOUP images.
\end{abstract}

[Submitted to Solar Physics] 